\title{
ARTEMIS: Neutralizing BGP Hijacking within a Minute
}

\author{
Pavlos Sermpezis \\ ICS-FORTH \\ sermpezis@ics.forth.gr \\ Xenofontas \\ Dimitropoulos \\ ICS-FORTH and University of \\ Crete \\ fontas@ics.forth.gr
}

\author{
Vasileios Kotronis \\ ICS-FORTH \\ vkotronis@ics.forth.gr \\ Danilo Cicalese \\ CAIDA, UC San Diego / Telecom \\ ParisTech \\ cicalese@enst.fr
}

\author{
Petros Gigis \\ ICS-FORTH \\ gkigkis@ics.forth.gr \\ Alistair King
CAIDA, UC San Diego
alistair@caida.org
}

\author{
Alberto Dainotti \\ CAIDA, UC San Diego \\ alberto@caida.org
}

\section{ABSTRACT}

BGP prefix hijacking is a critical threat to Internet organizations and users. Despite the availability of several defense approaches (ranging from RPKI to popular third-party services), none of them solves the problem adequately in practice. They suffer from: (i) lack of detection comprehensiveness, allowing sophisticated attackers to evade detection, (ii) limited accuracy, especially in the case of third-party detection, (iii) delayed verification and mitigation of incidents, reaching up to days, and (iv) lack of privacy and of flexibility in post-hijack counteractions, from the side of network operators. In this work, we propose ARTEMIS, a defense approach (a) based on accurate and fast detection operated by the AS itself, leveraging the pervasiveness of publicly available BGP monitoring services and their recent shift towards real-time streaming, thus (b) enabling flexible and fast mitigation of hijacking events. Compared to previous work, our approach combines characteristics desirable to network operators such as comprehensiveness, accuracy, speed, privacy, and flexibility. Finally, we show through real-world experiments that, with the ARTEMIS approach, prefix hijacking can be neutralized within a minute. Based on work
\end{abstract}

Permission to make digital or hard copies of part or all of this work for personal or classroom use is granted without fee provided that copies are not made or distributed for profit or commercial advantage and that copies bear this notice and the full citation on the first page. Copyrights for third-party components of this work must be honored. For all other uses, contact the owner/author(s).

ANRW'18, July 16, 2018, Montreal, QC, Canada

(C) 2018 Copyright held by the owner/author(s).

ACM ISBN 978-1-4503-5585-8/18/07.

https://doi.org/10.1145/3232755.3234554 under submission to IEEE/ACM Transactions on Networking. Sermpezis, P. and Kotronis, V. and Gigis, P. and Dimitropoulos, X. and Cicalese, D. and King, A. and Dainotti, A.; "ARTEMIS: Neutralizing BGP Hijacking within a Minute", https://arxiv.org/abs/1801.01085v3

ACM Reference Format:

Pavlos Sermpezis, Vasileios Kotronis, Petros Gigis, Xenofontas Dimitropoulos, Danilo Cicalese, Alistair King, and Alberto Dainotti. 2018. ARTEMIS: Neutralizing BGP Hijacking within a Minute. In ANRW '18: Applied Networking Research Workshop, July 16, 2018, Montreal, QC, Canada. ACM, New York, NY, USA, 1 page. https://doi.org/10.1145/3232755.3234554 